\title{
ORIGINALES
}

\section{Evolución del concepto calidad y aporte al desarrollo regenerativo desde la estrategia empresarial}

HÉCTOR CANOSSA MONTES DE OCA*

* Doctor en Ciencias Empresariales. Universidad Nacional, Guanacaste, Costa Rica. E-mail: hector.canossa.montesdeoca@una.ac.cr. ORCID: 0000-0003-0952-3193. Google Scholar: https://scholar.google.com/citations?hl=en\&user=k2GDK4EAAAAJ. 


\section{COMO CITAR ESTE ARTÍCULO}

How to cite this article:

Canossa Montes de Oca, H. (2021). Evolución del concepto calidad y aporte al desarrollo regenerativo desde la estrategia empresarial. Revista Perspectiva Empresarial, 8(2), 48-64.

Recibido: 07 de julio de 2021 Aceptado: 08 de octubre de 2021
RESUMEN Objetivo. Relacionar la calidad en las empresas con el aporte al desarrollo regenerativo; específicamente se busca describir el concepto actual de calidad, su relevancia y relación con la sostenibilidad y la estrategia de las empresas. Metodología. Se realizó una revisión documental con propuestas para incentivar el desarrollo regenerativo desde la estrategia de las organizaciones. Resultados. Se proponen aportes potenciales de la calidad al desarrollo regenerativo y se valora la calidad como mezcla de satisfacción, percepción y expectativas al revisar sus beneficios y costos en una organización. Conclusiones. Los aportes de la calidad se pueden orientar a acciones estratégicas desde una cadena de abastecimiento sostenible para la creación y promoción de productos reutilizados, reciclados o regenerados, el apoyo a políticas regenerativas, la comercialización de productos verdes y finalmente el aumento del capital humano formado y comprometido con el desarrollo regenerativo.

PALABRAS CLAVE empresa, estrategia, calidad, sostenibilidad, desarrollo regenerativo.

\section{Evolution of the concept of quality and business strategy contribution to regenerative development}

ABSTRACT Objective. To establish a relationship between quality and contribution to regenerative development in companies; specifically, to describe the current concept of quality, its relevance and relationship with sustainability and company strategy. Methodology. A documentary review was conducted with proposals to encourage regenerative development through organizational strategy. Results. Potential contributions of quality to regenerative development are proposed and quality is assessed as a combination of satisfaction, perception and expectations in an organization by reviewing its benefits and costs. Conclusions. The contributions of quality can be oriented to strategic actions such as a sustainable supply chain for the creation and promotion of reused, recycled or regenerated products; support to regenerative policies; commercialization of green products; and an increase in trained human capital committed to regenerative development.

KEYWORDS Company, strategy, quality, sustainability, regenerative development. 


\section{Evolução do conceito de qualidade e contribuição para o desenvolvimento regenerativo a partir da estratégia empresarial}

RESUMO Objetivo. Relacionar a qualidade nas empresas com a contribuição para o desenvolvimento regenerativo; especificamente, descrever o conceito atual de qualidade, sua relevância e relação com a sustentabilidade e a estratégia da empresa. Metodologia. Foi realizada uma revisão documental com propostas para incentivar o desenvolvimento regenerativo a partir da estratégia das organizações. Resultados. As potenciais contribuições de qualidade para o desenvolvimento regenerativo são propostas e a qualidade é avaliada como uma mistura de satisfação, percepção e expectativas, revendo seus benefícios e custos em uma organização. Conclusões. As contribuições de qualidade podem ser orientadas para ações estratégicas a partir de uma cadeia de abastecimento sustentável para a criação e promoção de produtos reutilizados, reciclados ou regenerados, o apoio a políticas regenerativas, a comercialização de produtos verdes e, finalmente, o aumento do capital humano treinado e comprometido com o desenvolvimento regenerativo.

PALAVRASCHAVE empresa, estratégia, qualidade, sustentabilidade, desenvolvimento regenerativo. 


\section{Introducción}

El crecimiento del mundo globalizado en múltiples dimensiones desde la demografía, economía, tecnología y producción en general ha generado efectos negativos en los ecosistemas, lo que ocasiona daños sustanciales en el planeta; el cambio climático atenta contra el futuro de las generaciones, esto requiere acciones inmediatas y continuas no solo para el crecimiento económico de las empresas y países sino para aportar a un desarrollo regenerativo que restaure los elementos del entorno a través de organizaciones que con acciones estratégicas regenerativas devuelvan más a la sociedad y al planeta de lo que obtienen de ella (Llain y Hawkins, 2020; Radjou, 2020).

El mundo ante las revoluciones industriales ha sufrido consecuencias que atentan contra el futuro de la sociedad, por esto se requieren esfuerzos para conservar recursos y preservar el entorno para la vida. Se debe buscar una regeneración del ambiente con iniciativas basadas en desarrollo sostenible, economía circular y desarrollo regenerativo que más que conceptos deben convertirse en acciones reales para lograr impactos positivos en un planeta desgastado. La Agencia de Transición Ecológica ADEME - de Francia define siete campos de acción de la economía circular: un suministro sostenible; el diseño ecológico; ecología industrial y territorial; sistema producto-servicio; consumo responsable; incremento de la duración del uso y el reciclaje.

\section{Müller (2016) explica que}

\section{hemos conducido un desarrollo degenerativo con elevadas tasas de extracción del capital natural para satisfacer una cultura de consumo suntuoso que ha favorecido únicamente a unos pocos, parece que el ser humano se ha olvidado que el mundo natural es el que mantiene toda vida en el planeta. (p. 2)}

Esta gestión de recursos a nivel mundial y las decisiones políticas, económicas y empresariales ha empeorado el cambio climático que genera efectos como las alteraciones en el aumento de $\mathrm{CO}_{2}$ y pérdidas de biodiversidad, desastres naturales como incendios forestales e inundaciones, así como cambios en los océanos y en los suelos que atentan con la supervivencia futura.
Como contraparte, el concepto de desarrollo regenerativo se basa en los seis pilares del desarrollo sostenible (político, económico, espiritual, ecológico, cultural y social), "con un enfoque no sólo en frenar la degradación de los mismos y más bien en la regeneración en cada sector, que es más allá de la reconstrucción o restauración e implica una mejora permanente de éstos" (Müller, 2016, p. 24). El paradigma de la sostenibilidad regenerativa se orienta a una visión del mundo ecológica que reconceptualiza las relaciones entre los sistemas tecnológicos, ecológicos, económicos, sociales y políticos del ser humano; este paradigma regenerativo busca el compromiso con un mundo vivo y con una visión ecológica y asociación cocreativa con la naturaleza basada en estrategias de adaptación, resiliencia y regeneración (Zhang, 2014; du Plessis, 2011).

Esta investigación pretende relacionar la evolución del concepto de calidad y su aporte para el desarrollo regenerativo desde el punto de vista de la estrategia empresarial. Los objetivos específicos incluyen: describir el concepto de calidad para las organizaciones actuales; identificar antecedentes que muestren la importancia de la calidad; reconocer los conceptos de satisfacción, percepción, expectativas y su relación con la calidad de las empresas; valorar diversos beneficios y costos que implican la aplicación o no de la calidad y considerar los principios para la gestión de la calidad en una organización que permiten aportes valiosos al desarrollo regenerativo por parte de las empresas.

La calidad debido a la alta competitividad y al comportamiento de los clientes e interesados en una organización o producto se puede definir como el conjunto de características, servicio, proceso, organización - o incluso una personaque se basan en la capacidad para satisfacer las necesidades y expectativas del cliente, usuario e interesados con relación a lo que percibe y cumplir las especificaciones con la que fue diseñado o propuesto.

Los conceptos de calidad cambiaron gradualmente desde el logro de los estándares de calidad hasta un concepto ligado a la satisfacción de las necesidades y expectativas del cliente (Yang, 2017). Está calidad esperada debería incluir el componente facilitador del desarrollo sostenible. 
Paradigma global de las Naciones Unidas, definido en 1987 en el Informe Brundtland como "desarrollo que satisface las necesidades de la generación presente, sin comprometer la capacidad de las generaciones futuras de satisfacer sus propias necesidades" (p. 59). Entretanto, Camisón, Cruz y González (2006) exponen que "el elemento de «satisfacer necesidades», subyacente al concepto moderno de calidad, evidencia que el desarrollo sostenible se orienta hacia la conservación y mejora de la calidad del entorno natural" (p. 440).

El empresario hoy debe comprender que este concepto de calidad ha evolucionado hasta convertirse en una forma de gestión que implica la mejora continua, llevando al concepto hacia una gestión de calidad total; aplicable para cualquier organización y a todos los niveles de esta y que, además, afecta a todos los procesos y personas.

En este documento se buscan los aportes potenciales de la calidad para la sostenibilidad y la regeneración, generando consciencia en los gestores empresariales y las personas en general, desde las decisiones de consumo hasta las decisiones de producción u operaciones empresariales para entregar calidad y aportar a un desarrollo regenerativo a partir de la estrategia de las empresas.

\section{Fundamentos teóricos}

Desde el principio los humanos en su ambiente han requerido calidad (Lee, 2015), los cazadores y recolectores necesitaron herramientas que cumplieran su función para lograr alimentarse; en el origen del comercio y sus regulaciones comienzan mayores exigencias de las partes; las máquinas $\mathrm{y}$ avances tecnológicos hacen evolucionar un concepto que inicialmente implicaba la inspección de productos terminados mediante un control de calidad; luego se buscó la prevención a través del aseguramiento de calidad; a hoy se convierte en un sistema de gestión - que incluye toda la organización- centrado en el cliente, el valor y la excelencia en el que adquiere mayor importancia el bienestar de los colaboradores en el lugar de trabajo, así como la sostenibilidad.
Es así como inicia las estrategias de control de calidad total mediante programas diseñados para actuar frente a toda la organización empresarial. Se comienza a hablar de los sistemas de gestión de calidad total o Total Quality Management TQM- (Robledillo y Velázquez, 2013; Zehir and Müceldilli, 2012).

Asimismo, Cuatrecasas y González (2017) afirman que

$$
\begin{aligned}
& \text { la calidad sigue ampliando sus objetivos a todos } \\
& \text { los departamentos de la empresa, involucra a } \\
& \text { todos los recursos humanos liderados por la } \\
& \text { alta dirección y aplica desde la planificación y } \\
& \text { diseño de productos y servicios, que da lugar } \\
& \text { a una nueva filosofía de la forma de gestionar } \\
& \text { una empresa. (p. 16) }
\end{aligned}
$$

La calidad ha tenido muchas definiciones. Feigenbaum definía calidad en términos de la satisfacción del cliente y debido a las necesidades cambiantes de los clientes, la calidad es multidimensional y dinámica; Shewhart indicaba que la calidad tiene dos aspectos: el subjetivo (lo que quiere el cliente) y el objetivo (características físicas y mesurables de los bienes o servicios); por su parte, Deming afirmaba que la calidad es multidimensional y debe definirse en términos de la satisfacción del cliente (Summers, 2006).

Por otra parte se considera importante revisar acontecimientos históricos en la evolución del concepto de calidad. A través del tiempo con los avances globales la calidad ha tomado mayor relevancia hasta adquirir el potencial para aportar al desarrollo regenerativo con empresas más integrales y responsables con su entorno y las personas con las que tiene relación.

Al principio, la calidad se centraba en los productos terminados. En la segunda Revolución Industrial la exigencia en la fabricación masiva, las máquinas y la electricidad adquieren un papel clave en el crecimiento de las empresas (Patiño, 2019). La calidad se basó en la inspección del producto que realizaban los consumidores, los precios de los productos tendían a ser altos por la corta vida útil de las máquinas, no se daba tanta importancia a la eficiencia e existía una amplia división del trabajo y pocas exigencias de capacitación, pues se desconocían estas áreas de trabajo. 
Hacia 1900 Ford introdujo la producción en serie - masiva o en línea- en un espacio de trabajo organizado para la productividad en las tareas (Wilson, 2013), mejorando los tiempos a través de la llamada línea de ensamblaje, en este avance se hacen pruebas de errores e inspección dentro del proceso. Esto muestra un inicio del pensamiento sobre la productividad y la relevancia que se le comienza a dar a la estandarización y a la calidad (Palominos, 2007). Este aporte sigue vigente con ajustes de tecnología, pero siempre aprovechando las ventajas de línea para ser eficientes; Cuatrecasas (2009) plantea que "desde la primera cadena de montaje para ensamblar el modelo T en 1913, la tendencia ha sido ensamblar o montar en cadena en cualquier enfoque de gestión" (p. 395).

También se incluyeron los principios y métodos de gestión científica -homónimos del libro publicado en 1911 por Frederick Winslow Taylor en el que propone la administración científicaque buscan productividad, división del trabajo, mayor exigencia a los emplead os y enseñar a hacer bien las tareas; algunos de los principios de esta propuesta son el estudio de tiempos y movimientos, estandarización de herramientas, departamento de planificación de ventas, tarjetas de enseñanza para los trabajadores, reglas de cálculo para el corte de materiales, métodos de determinación de costos, selección de colaboradores por tareas e incentivos por terminar el trabajo a tiempo (Carlson, 1997; Carró y Caló, 2012; Grachev and Rakitsky, 2013).

Aquíla calidad se empezaba a enfocar no solo en los productos, sino también en los procesos. Ante la ineficiencia en la producción y falta de calidad Taylor descubrió que la producción y la paga eran precarias, asícomo la ineficiencia y el desperdicio prevalecían, concluyendo que las decisiones administrativas no eran sistemáticas y que no había investigación que determinara mejores formas de producción; por ello presentó una segunda perspectiva de la administración conocida como administración científica, en donde enunciaba la aplicación de métodos científicos para analizar el trabajo y la determinación de cómo llevar a cabo eficientemente la producción (Bateman y Snell, 2009).

Alrededor de 1920 se da el control estadístico de procesos o la calidad del producto, la inspección de grandes volúmenes de producto se hace de forma más eficiente a través de muestras de lo producido. Esto inició en 1924 en la empresa Bell, Walter Shewhart introdujo las gráficas de control a través de un diagrama sencillo que indicaba los límites superiores e inferiores de las tendencias de comportamiento de una tarea. En 1931, Shewhart entregó un fundamento científico a la calidad por medio del libro Economic Control of Quality of Manufactured Product. En este se dieron a conocer las cartas de control y el estudio de la calidad a través de variables, estableciendo que el conocimiento obtenido con la realización de estudios estadísticos puede usarse para mejorar el control mediante la estabilización y reducción de la variación del proceso (Gutiérrez, 2010).

Después de la Segunda Guerra Mundial se promovieron las estadísticas para el control y la mejora necesaria de la calidad. Aquí las organizaciones tuvieron cada vez menos tiempo y disponibilidad de recursos para fabricar y en el que no solo se requería calidad del producto, sino también del proceso; así, más que la inspección se requiere prevención. En este sentido Platas y Cervantes (2017) explican que "en 1946 se reunieron en Londres, delegados de 25 países con la finalidad de crear una nueva organización que facilitara la coordinación entre diferentes industrias al generar estándares o guías de carácter internacional" (p. 38). Este avance crea un consenso entre diversos profesionales en múltiples áreas, buscando promover la calidad a través de procesos estandarizados que cumplan con normas establecidas, permitiendo a organizaciones y negocios internacionales garantizarse calidad.

De igual manera hay que tener en cuenta la conceptualización de la mejora continua. William Deming viajóa Japón después de la Segunda Guerra Mundial para impartir diversos seminarios sobre la calidad, promoviendo el aseguramiento de esta con una propuesta de administración de la calidad, enfocándola en la medida que se gestiona la satisfacción del cliente. En su propuesta de gran importancia se tiene la comunicación, participación de los gerentes, el uso de la estadística para el análisis y la mejora continua. Deming (1986) también promovió y popularizó el denominado ciclo de Deming —o de mejora continua-que incluye las tareas de planificar, hacer, verificar o estudiar y actuar; este ciclo fue propuesto originalmente por Walter Shewhart como un método sistemático para la resolución de problemas. Deming describió 
su trabajo como una administración de la calidad y consideraba al consumidor como el factor más importante (Reid, Koljonen and Buell, 1999;
Summers, 2006; Díaz, Tamayo y Armijos, 2018). Por último, hay un surgimiento de publicaciones y organizaciones sobre la calidad (tabla 1).

Tabla 1. Publicaciones y organizaciones sobre la calidad

\begin{tabular}{cl}
\hline Avance histórico en la calidad & Descripción \\
\hline & En 1944 se publica la primera revista sobre control de calidad, \\
Ilamada Industrial Quality Control; en 1946 se funda la American \\
Srimera revista sobre control de calidad & y capacitaciones sobre calidad. \\
\hline
\end{tabular}

Unión Japonesa de Científicos e Ingenieros -JUSE-

\section{Manual de control de la calidad}

Sistema de Producción Toyota

Ishikawa y la cultura de calidad

Calidad total

Poka-Yoke
En 1946 se creó la Unión Japonesa de Científicos e Ingenieros -JUSE-, un grupo de profesionales de investigación para mejorar los procesos de control de calidad (Fisher, 2009); la JUSE, en 1951, establece el Premio Deming de la calidad.

En 1951 Joseph Juran publicó el Manual de control de la calidad para la formación de los responsables de calidad en organizaciones; considerando la importancia de presupuestary planificar el negocio, tener un control de costos y el control de inventario, buscando la mejora de beneficios para la organización.

En los años 50, ingenieros de Toyota (dirigidos por Taiichi Ohno) introdujeron diversas propuestas en la industria automotriz; teniendo éxito en la reducción de errores y costos, aportando al desarrollo de producción justo a tiempo; en términos generales, promoviendo el Sistema de Producción Toyota (Mishraa, Kumar and Garg, 2013).

En 1957, Kaoru Ishikawa da relevancia a la promoción de una cultura de calidad en todo el funcionamiento de una organización; por esto mismo propone iniciativas como los círculos de calidad (reuniones de trabajadores para procesos de mejora) o el reconocido diagrama de Ishikawa o diagrama de causa-efecto, útil para la resolución de problemas y mejora de procesos; el papel de los colaboradores es base para lograr cero defectos (Ishikawa, 1986).

En 1961, Armand Feigenbaum publica Total Quality Control en el que se propone el concepto de calidad total. Para Ishikawa (1986) el concepto de control total de la calidad según Feigenbaum es "un sistema eficaz para integrar los esfuerzos en materia de desarrollo de calidad, mantenimiento de calidad y mejoramiento de calidad realizados por los diversos grupos en una organización, de modo que sea posible producir bienes y servicios a los niveles más económicos y que sean compatibles con la plena satisfacción de los clientes” (p.112).

En los años 60 el ingeniero Shigeo Shingo introduce en Toyota la herramienta Poka-Yoke (Poka se traduce como errores imprevistos y Yokeru como acción de evitar). Esto se conoce como "a prueba de errores" y busca el diseño de procesos, evitando equivocaciones humanas o automatizadas. Tiene como funciones controlar o advertir, por ejemplo: a través de formas, colores o sonidos como alarmas (el uso conocido del color rojo en botones para indicar la importancia de este, alarmas en caso de presión en una máquina entre otros)(Zhang, 2013). 


\begin{tabular}{|c|c|}
\hline Avance histórico en la calidad & Descripción \\
\hline Despliegue de la Función de la Calidad -DFC— & $\begin{array}{l}\text { En 1972, Yoji Akao desarrolla el Despliegue de la Función de la Calidad } \\
\text {-DFC- en un astillero de la empresa Mitsubishi en Japón. El DFC } \\
\text { empieza por estudiary escuchar a los clientes a través de estudios de } \\
\text { mercado en busca de un producto cada vez mejor; la realimentación } \\
\text { de los clientes es útil para la toma de decisiones de ingeniería, diseño } \\
\text { y mercadeo (Akao and Mazur, 2003). }\end{array}$ \\
\hline Fundación Europea para la Gestión de la Calidad & $\begin{array}{l}\text { En } 1988 \text { se crea la Fundación Europea para la Gestión de la Calidad } \\
\text {-EFQM-para promover la competitividad de las empresas europeas; } \\
\text { la calidad se enfoca en el poder que tienen los consumidores y se } \\
\text { visualiza como parte de la supervivencia de la organización. }\end{array}$ \\
\hline Normas ISO 9000 & $\begin{array}{l}\text { En el año } 2000 \text { la Organización Internacional de Normalización difundió } \\
\text { los documentos de las normas ISO 9000:2000, ISO 9001:2000 e ISO } \\
\text { 9004:2000 que consideran los principios de un sistema de gestión } \\
\text { de la calidad y las directrices para la mejora del desempeño. Estas } \\
\text { normas permanecen en constante actualización, la primera edición de } \\
\text { las normas ISO 9ooo se publicó en } 1987 \text { (García, Quispe y Ráez, 2001). }\end{array}$ \\
\hline "The Toyota Way" & $\begin{array}{l}\text { En el año } 2001 \text { Toyota resume su filosofía, valores e ideales de } \\
\text { fabricación y publica The Toyota Way que se enfoca en áreas clave } \\
\text { de la mejora continua y el respeto por las personas (el cual desde } \\
\text { los años } 50 \text { venía implementando con su TPS - Toyota Production } \\
\text { System-). Este amplía temas como el sistema justo a tiempo (Just in } \\
\text { Time), muda (eliminación de desperdicios) o la metodología } 5 \text { s (que } \\
\text { incluye clasificar, ordenar, limpieza, estandarizary la disciplina para } \\
\text { un entorno de trabajo eficiente) (Liker, 2003). }\end{array}$ \\
\hline
\end{tabular}

Fuente: elaboración propia por parte del autor.

Existen muchos más aportes y personajes en el campo de la calidad, esta breve compilación busca reflejar los esfuerzos que se han hecho en el tema y cómo va evolucionando de acuerdo con los cambios y necesidades de los mercados. Por consiguiente cuando un empresario pretende la satisfacción del cliente, su lealtad, así como una empresa que mejore continuamente y aporte al desarrollo regenerativo, puede considerar los principios que se proponen en las normas ISO; estos incluyen el enfoque al cliente, liderazgo orientado al propósito común, el compromiso de las personas, el enfoque en procesos, el enfoque en la mejora continua, la toma de decisiones basada en la evidencia y finalmente la gestión de relaciones que mejoran las condiciones de trabajo y la entrega de calidad.

Las empresas deben transmitir estos avances en la aplicación de la calidad hacia el desarrollo de un sistema que interrelacione todas las actividades empresariales con el entorno, preocupándose por la mejora continua no solo de la empresa sino de los pilares del desarrollo sostenible.

\section{Summers (2006) afirma que}

$$
\begin{aligned}
& \text { para satisfacer de manera óptima las } \\
& \text { necesidades, requerimientos y expectativas } \\
& \text { del cliente, las organizaciones eficientes } \\
& \text { crean y utilizan sistemas de calidad. Los } \\
& \text { sistemas de administración de la calidad } \\
& \text { conjuntan los ingredientes necesarios para } \\
& \text { quelos empleados dela organización puedan } \\
& \text { identificar, diseñar, desarrollar, producir, } \\
& \text { entregar y apoyar los productos y servicios } \\
& \text { que el cliente desea. (p. 35) }
\end{aligned}
$$

Así pues, la gestión de la calidad apoya los enfoques para el desarrollo sostenible (Siva et al., 2016). A través del tiempo la producción ha aumentado exponencialmente; muchos de los 
exponentes de la calidad se centraron en cómo mejorar la eficiencia y aportar al crecimiento y rentabilidad de las empresas, pero falta darle más relevancia al ambiente externo y el impacto que genera en el entorno. Hay esfuerzos como la familia ISO 14000 sobre gestión ambiental, ISO 50001 de gestión energética o la norma ISO 14021 sobre etiquetas y declaraciones medioambientales; además de metodologías de proyectos como PRiSM ${ }^{\mathrm{TM}}$ del Green Project Management y los Objetivos de Desarrollo Sostenible -ODS - promovidos desde la Organización de las Naciones Unidas; lo anterior, es un impulso hacia una mayor implementación de prácticas de desarrollo regenerativo desde la estrategia de las empresas sin importar el sector o tamaño de la organización.

\section{Metodología}

El presente artículo se basa en una metodología cualitativa, de tipo documental, con un alcance exploratorio, llevando a cabo una revisión literaria e incluyendo propuestas para promover el desarrollo regenerativo desde la estrategia empresarial. Para este propósito se hicieron consultas de fuentes teóricas en repositorios tales como Scopus, SciELO, Dialnet, Emerald y ProQuest.

El diseño empleado en la investigación fue no experimental-exploratorio en el que se efectúa una exposición narrativa que detalla los elementos relacionados a la calidad, el desarrollo regenerativo y cómo desde las decisiones empresariales y estrategias se puede aportar en este campo. Las etapas del trabajo incluyen la revisión documental para la orientación de la evolución del concepto de calidad y posteriormente como segunda etapa las propuestas hacia el desarrollo regenerativo y la utilización de la calidad en la estrategia empresarial para las mejoras en la gestión empresarial.

\section{Resultados y discusión}

\section{Propuesta de los aportes potenciales de la calidad al desarrollo regenerativo}

El concepto de calidad se ha hecho holístico y estratégico, pues integra las diversas áreas de la empresa y va más allá del componente económico y de consumo; por ello su potencial para mejorar las formas degestión en las empresasy orientarlas a que sean más responsables con el entorno, generando empresas que aporten al desarrollo regenerativo. Algunos de estos aportes considerados se muestran en la figura 1.

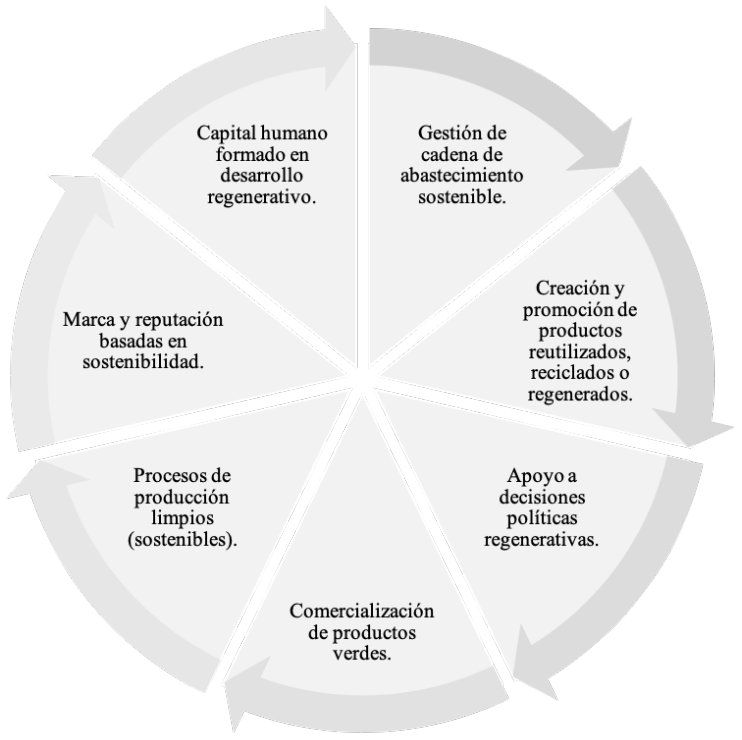

Figura 1. Aporte potencial de la calidad al desarrollo regenerativo. Fuente: elaboración propia por parte del autor.

En una gestión de la cadena de abastecimiento comprometida con la sostenibilidad las relaciones con proveedores y adquisición de materiales debe aportar a que no haya un deterioro o desgaste de recursos que impacten en el ambiente y buscar medidas de mitigación o restauración; para esto la calidad juega un papel fundamental en el control y mejora de procesos y relaciones en la cadena de suministro.

De igual manera la creación y promoción de productos que han atravesado procesos como 
el reciclado y/o promover la reutilización de materiales como las iniciativas de logística inversa que devuelven productos o partes a la cadena de producción, permitiendo reducir desechos o productos de un solo uso.

Reducir, Reusar y Reciclar eran los conceptos conocidos, basados en las $3 \mathrm{R}$ del ciclo de vida económico, a los que se agregaron 3 conceptos más. Estos integran la definición de economía circular, a través de: recuperar los materiales, remanufacturar los productos, y rediseñar las tecnologías. (Valenzuela, Espinoza y Alfaro, 2019, p. 312)

A la vez el apoyo y promoción de decisiones políticas hacia la creación de leyes o iniciativas que permitan proteger los recursos, por ejemplo: recursos marítimos; creación de áreas de conservación; la no utilización de plástico de un solo uso y legislación para industrias agrícolas que generan contaminación o deterioro de los suelos. Estas decisiones deben abordarse tanto en gobiernos centrales como en locales y las empresas deben participar y comprometerse en este proceso.

También hay que tener en cuenta el aprovechamiento de oportunidades de comercialización de bienes o servicios ecológicos, aquí las empresas deben identificar los segmentos generacionales que están comprometidos con la situación del planeta y aprecian las iniciativas verdes; esto puede ser un componente estratégico a usarse en el marketing, en donde clientes e involucrados de la empresa puedan apreciar que la calidad también se basa en su aporte hacia la regeneración de los factores del entorno.

El término verde se ha utilizado para productos que procuran seguir una producción y consumo sustentable, algunos aspectos según la norma ISO 14021 incluyen: que sea reciclable; que utilice material reciclado; energía recuperada; que sea reutilizable o recargable; susceptible de ser descompuesto en 'compost' (abono); que aporte a la reducción de los residuos sólidos; conserva o ahorra energía; que es eficiente con el agua, conserva o ahorra; que reduce la utilización de recursos; que es degradable, biodegradable o fotodegradable; desmontable o bien tiene la aprobación por un grupo en particular o apoyo de una causa medioambiental (Martínez et al., 2018).
Otro de los puntos importantes es la ecoeficiencia en la gestión y los procesos de producción, administrando negocios con criterios medioambientales y de desarrollo humano. Se rompe con las prácticas de producción convencionales para satisfacer un mercado con recursos naturales y materias primas no renovables sino, más bien, "maximizar el beneficio económico y el cuidado del medio ambiente" (Ponce y Loor, 2020, p. 254).

Por ello las empresas deben enfatizar en el uso de energías limpias, protección del agua y alimentos, capacitarse e integrar tecnologías basadas en movilidad, accesibilidad y conveniencia; sacando provecho de los avances en robótica, inteligencia artificial y realidad virtual que podrían aportar a una cultura ecológica y regenerativa para el bienestar humano.

Por otro lado, estratégicamente las empresas ahora cuidan más su marca y reputación dado que la Internet y las redes sociales permiten que la información fluya de manera más rápida; operacionalmente hay un incremento de empresas en red, mucha influencia tecnológica hacia la eficiencia, cadenas de valor mucho más ajustables y el uso de empresas plataforma. De ahí que la responsabilidad de las empresas grandes y líderes de los mercados sea fundamental para lograr el desarrollo regenerativo por ser influencers ecológicos.

Así, en áreas de gestión de personas, la calidad puede aportar en la creación de nuevos perfiles de trabajo y entornos laborales más flexibles para fortalecer las relaciones, fomentar la creatividad y mejorar los sistemas de compensación; esto entre otros elementos que valoren a los humanos en la empresa, desarrollen talento y crear así ventajas competitivas, lo que aportaría a una regeneración del componente social y económico del entorno. Además se debe fomentar un capital humano con consciencia ambiental y con oportunidades de formación en estas áreas (por ejemplo, con carreras universitarias o capacitación sobre sostenibilidad, gestión ambiental y desarrollo regenerativo). 


\section{La calidad como mezcla de satisfacción, percepción y expectativas}

Las organizaciones a través del mercadeo comunican a los clientes y crean expectativas; cuando el cliente tiene el contacto con la organización tendrá una percepción sobre lo que recibe. Esta relación entre expectativas y percepción definen la satisfacción: si se cumplen las expectativas se tendrá un cliente satisfecho, se deberá buscar superar las expectativas para que el cliente pueda recordar la organización y aumentar las posibilidades de recomendarla.

Parasuraman, en el libro Calidad total en la gestión de servicios, en busca de mejorar la calidad de servicio analizaba las palabras expectativa y percepción; con estas dos palabras se hace referencia a que la satisfacción del cliente puede medirse a través del planteamiento de una sencilla ecuación en la que la satisfacción es igual a la percepción menos la expectativa (Galarreta, 2019).

Esta percepción está ligada al valor que genera la empresa para el cliente. Para que las organizaciones generen valor sean competitivas y logren clientes satisfechos, leales y entusiastas deben conocer qué necesita, desea y espera de los productos, procesos y personas de la empresa; asimismo, conocer las empresas competidoras permite diseñar mejores productos y estrategias oportunas. Para este conocimiento y mejor entrega de calidad es necesaria "la investigación de mercado y los SIM son importantes para hacer un marketing efectivo, posicionar una empresa, producto o marca frente a la competencia y lograr ejecutar una planificación estratégica eficaz" (Canossa, 2019, p. 47).

En el servicio la empresa puede orientar al cliente hacia iniciativas que aportan al desarrollo regenerativo, cumpliendo con las expectativas y agregando valor. El enfoque orientado al cliente para generar valor implica factores tales como la entrega deinformación suficiente, esto es fundamental para que el cliente no tenga incertidumbre mientras se encuentra en una empresa. Además los aspectos percibidos por los sentidos al hacer referencia a elementos visuales, sonidos, olores, cosas que se pueden tocar en el sitio (por ejemplo, la apariencia física del lugar como su decoración, la calidad de los objetos, la limpieza y el orden) son aspectos que pueden influir en la satisfacción.

La empatía también es importante para las personas, la sinceridad del prestador del servicio y su capacidad para analizar al cliente y ponerse en su lugar para comunicarse y dar un trato óptimo al aportar personalización. Cabe agregar la capacidad de respuesta y garantía, en donde los colaboradores de la empresa puedan responder rápido y orientar de la mejor manera con garantías adecuadas; además, si es un problema generado por el cliente, brindar el acompañamiento e información más oportuna para la resolución.

Finalmentela gestión de la calidad en el servicio genera la oportunidad de fomentar la orientación de empresa regenerativa como propuesta de valor, ya que el cliente percibirá la responsabilidad de la empresa con el ambiente y su búsqueda por aportar a la restauración o contribución a factores del entorno tales como los aspectos sociales o ecológicos.

\section{Beneficios de la calidad en una organización}

Buscar la mejora continua y la calidad en una organización promoverá que los clientes inviten a otros clientes. Además de prevenir problemas de calidad tales como pérdida de reputación, otorgar garantías e incluso implicaciones y costos legales; así pues, la calidad aporta para evitar conflictos y mejorar el bienestar de todos los involucrados.

La calidad promueve la satisfacción de los clientes e interesados, por lo que se convierte en un aspecto fundamental para la competitividad y sobrevivencia en el mercado. Por ello la calidad debe ser un elemento orientado también a la sostenibilidad y al desarrollo regenerativo, mejorando el entorno y aportando a la economía circular; siendo esta un modelo que busca una gestión de calidad reflejada en el procedimiento eficiente y eficaz de la planificación, organización, dirección y control de un ente económico con responsabilidad social y ambiental (Moscoso, Rojas y Beraún, 2019).

Los beneficios de la gestión de la calidad incluyen la reducción de costos, lo que evita ciertas acciones correctivas como son: retrabajos; 
desperdicios; racionalizando y aprovechando espacios; menores costos financieros por reducción de inventario a través de un mejor control (con ello se evitan devoluciones y quejas); asimismo, con la prevención, se reduce la posibilidad de averías en máquinas. De igual manera toda empresa que busca calidad puede incentivar mejoras en el clima de trabajo; de ahí que una empresa más social en la que se promueva compromiso y motivación mejora las condiciones de seguridad e higiene, promoviendo la disminución de accidentes laborales.

Por su parte en el ámbito externo también mejora las relaciones con los clientes y proveedores con más control, comunicación efectiva, menos conflictos y mayor estandarización que reduce la incertidumbre. La empresa contribuye con el entorno al buscar el desarrollo regenerativo desde el conocimiento de las necesidades presentes en su contexto y con la toma de decisiones estratégicas que aporten en este campo.

La calidad debe ser gestionada desde un punto de vista estratégico, es decir, considerando todas las decisiones y acciones de la organización y a todas las personas involucradas; en la figura 2 se muestran diversos aspectos involucrados y propuestos para considerar si se desea una organización gestionada con calidad total.

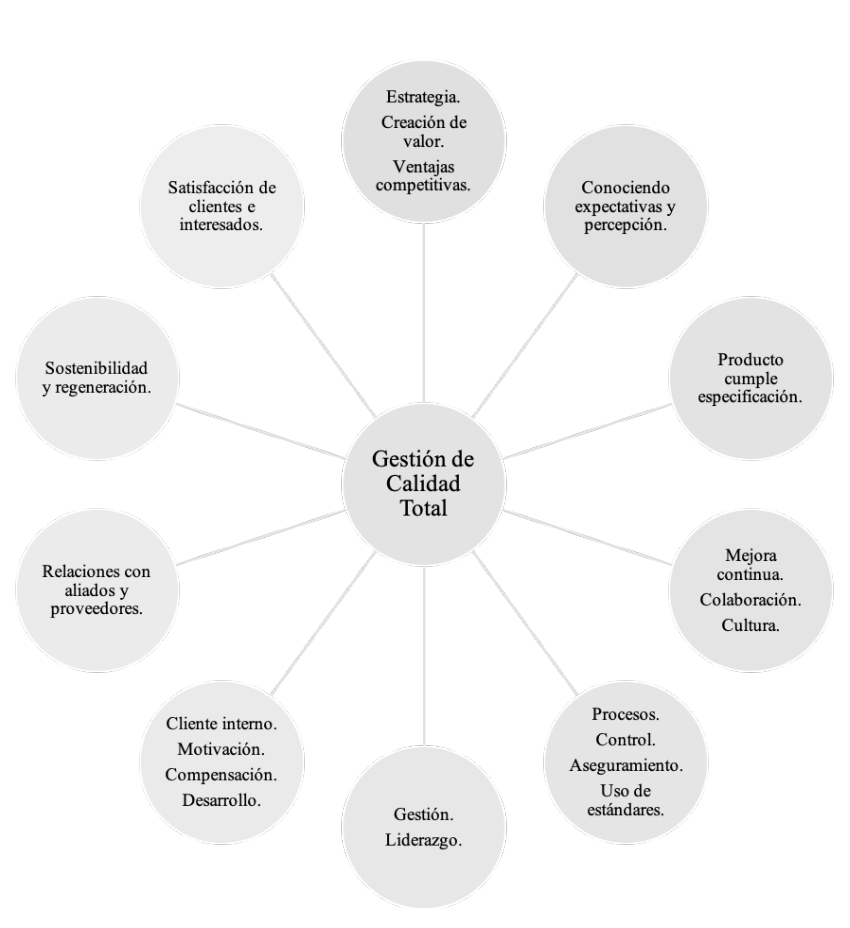

Figura 2. Aspectos involucrados propuestos a considerar para una gestión con calidad total. Fuente: elaboración propia por parte del autor.

Resulta importante mencionar que una empresa que entrega calidad total estará estrechamente ligada al concepto de responsabilidad social empresarial; en donde la conceptualización más holística y progresista expone que una empresa socialmente responsable valora el impacto de sus acciones en las comunidades, los trabajadores y en el medioambiente e incorpora efectivamente sus intereses en sus procesos y resultados. Además ejerce un especial respeto por las regulaciones y leyes que la sociedad considera legítimas,

pero también respeta los acuerdos y tratados internacionales sobre fiscalidad, prevención de la corrupción, respeto a los derechos humanos y derechos laborales, protección del medio ambiente y busca garantizar que cumplan estas regulaciones y principios, los subcontratistas, socios comerciales, 
proveedores y cualquier otro con quien realice negocios. (Departamento de Estudios, 2005, p. 8)

Una empresa que busca entregar calidad total comprende que la actividad empresarial no solo se centra en lucrar y en las exigencias del mercado sino que entiende que la empresa es parte de un entorno mucho más extenso y que debe aportar al futuro para un desarrollo económico, humano y en el mejor de los casos regenerativo.

Este concepto también busca promover desde las empresas los ecoemprendimientos ya sea dentro de la organización o generando nuevos negocios que crean valor social al crear cambios positivos en la calidad de vida de los individuos, aportando soluciones a los efectos secundarios indeseados en la producción o uso de bienes y servicios; pues "el gran deterioro ecológico que se padece actualmente en el planeta [...] pone en riesgo la supervivencia" (Arroyave y Marulanda, 2019, p. 170).

Las iniciativas que lleve a cabo la empresa hacia la gestión de la calidad total y aportes al desarrollo regenerativo son estratégicas, ya que buscan generar ventajas competitivas; y esto se debe traducir en atracción y retención de clientes, así como en valor agregado para los involucrados, por ello es importante considerar los costos de brindar o no calidad.

\section{Costos relacionados con la calidad}

En la gestión de calidad hay costos de preparación o prevención, costos de evaluación, así como los costos en los que se incurre por no entregar calidad y que pueden ser por fallos internos o externos (Omachonu, Suthummanon and Einspruch, 2004). Crosby (1987) resalta que "lo que cuesta dinero son las cosas que no tienen calidad - todas las acciones que resultan de no hacer bien las cosas a la primera vez" (p. 9).

Los costos de prevención o preparación son para evitar errores e implicarán que los colaboradores respondan y hagan bien sus tareas desde la primera vez, lo cual se reflejará en el resultado que entregan. Además, en estos costos de prevención, se incluye la evaluación que se visualiza en la producción ya terminada y auditorías de procesos para medir la conformidad con criterios y procedimientos preestablecidos por la empresa.

Algunos generadores de costos y que permiten calidad a través de la prevención son los colaboradores dedicados a la calidad de la empresa, las revisiones de diseño, las actividades de capacitación y la sensibilización en diversas áreas incluidas entre otras: la degestión hacia el desarrollo regenerativo; los mantenimientos preventivos; la evaluación de proveedores; encuestas a clientes sobre calidad ya sea de productos, atención al cliente o procesos específicos; así como costos generados por la implementación de sistemas de mejora continua.

También existen costos por no entregar calidad, conocidos como costos de la mala calidad - CMC-, en este punto se consideran dos escenarios: cuando el producto aún no lo ha recibido el cliente externo o cuando lo recibe y no es aceptado porque no cumple con las expectativas. Los costos internos se analizan como errores de la empresa detectados antes de que el producto sea aceptado por el cliente; esto se traduce en salidas de dinero para la empresa por el desperdicio de materiales, la refabricación, reinspección necesaria, pruebas repetitivas o incluso por si se debe desechar por completo el producto (Valenzuela, 2016).

Cuando el cliente percibe el error - y lo que le entregan es inaceptable-generalmente es una consecuencia más negativa para la empresa puesto que esto influye en su imagen, aunado a los efectos molestos para el cliente que afectan aún más su percepción tales como largas esperas para recibir una garantía o el efecto que se puede tener en el punto de venta por tener un cliente insatisfecho. Asimismo, esta imagen negativa que se lleva el cliente es probable que lo transmita a su grupo de influencia.

Cualquiera salida de dinero que puede ser consecuencia de no entregar calidad son las generadas al entregar garantías y hacer devoluciones, aplicar descuentos, la sustitución de productos defectuosos, indemnizaciones, la pérdida de clientes e incluso los daños al medioambiente (Arango, 2010). Cabe destacar que estos aspectos también implican el tiempo de trabajo de los colaboradores en aras de compensar y solucionar 
la situación, tiempo que podrían utilizar en nuevas ventas u otros procesos rentables para la empresa.

Con las mejoras continuas de calidad se decrecen los costos, posteriormente la empresa mejora en productividad al adquirir ventajas en el mercado y así estos dos elementos aportan a que la empresatenga mayor permanencia; esto es conocido como la cadena virtuosa de Deming, mostrando la importancia de gestionar de manera óptima la calidad en la empresa como elemento estratégico (Valenzuela, 2016). Además, la sustentabilidad es un dominio importante y legítimo de la calidad que exige la redefinición del valor para incorporar los costos sociales y ambientales; es una visión hacia el desarrollo regenerativo y la sustentabilidad, así al implementar calidad en la empresa se tendrá una repercusión en sus costos y eficiencia (Mortimer et al., 2018).

\section{Conclusiones}

Ban Ki-moon, en 2007, siendo Secretario General de las Naciones Unidas, expuso que "el futuro está en nuestras manos, juntos, debemos asegurarnos de que nuestros nietos no tendrán que preguntarnos por qué no logramos hacer lo correcto dejándoles sufrir las consecuencias" (como se citó en González, 2017, p. 266). De ahí que como seres humanos y desde las empresas como elemento estratégico se deben considerar con responsabilidad los negocios regenerativos, es decir, aquellos que mejoran y prosperan los sistemas sociales y ecológicos (Hahn and Tampe, 2020).

Este artículo genera una reflexión a través de una revisión literaria que aborda la evolución del concepto de calidad y su aporte para el desarrollo regenerativo. Se concluye que el concepto de calidad engloba más que productos que satisfacen necesidades y deseos de las personas a una calidad total en términos holísticos desde la empresa, sus integrantes, los clientes y la sociedad y deberá al mismo tiempo velar por la sostenibilidad del entorno y aportar al desarrollo regenerativo que requiere el planeta. La literatura muestra que las principales prácticas y programas de calidad asociados con el paradigma de la gestión de la calidad facilitan la adopción de prácticas ambientales en las empresas (Allur et al., 2018).

Los antecedentes en la revisión literaria muestran la importancia de la calidad en la que la empresa requiere líderes capaces de tener una visión estratégica inteligente y preocupada por el entorno, valorando los beneficios y costos que implican la aplicación de la calidad en empresas regenerativas; así pues, estos aportes pueden incluir entre otros: la gestión de cadena de abastecimiento sostenible; creación y promoción de productos reutilizados, reciclados o regenerados; consciencia y apoyo a decisiones políticas regenerativas; comercialización de productos verdes; procesos de producción limpios (sostenibles); marca y reputación basadas en sostenibilidad y capital humano formado en desarrollo regenerativo. La necesidad de regeneración es tan real como la degradación ecológica y cultural que se está dando en todo el planeta, por ello se requiere la creación de empresas regenerativas con abundancia multicapital (capital social, material, vivo, intelectual, experiencial, cultural y espiritual) para las generaciones venideras (Roland y Landua, 2017).

Finalmente debe quedar en nuestra mente que el término calidad para el desarrollo regenerativo debe promoverse tanto a nivel individual, empresarial, de gobiernos locales, gobiernos nacionales, regiones y el mundo que involucre decisiones y acciones continuas para renovar, restaurar y mejorar ecosistemas y los diversos pilares del desarrollo sostenible, incrementando las posibilidades a futuro dado que la estrategia empresarial y la competitividad no solo se verá determinada por un crecimiento económico sino por el aporte que deja la empresa en el entorno y para el futuro.

\section{Referencias}

Akao, Y. and Mazur, G. (2003). The leading edge in QFD: past, present and future. International Journal of Quality \& Reliability Management, 20(1), 20-35. 
Allur, E. et al. (2018). Quality and Environmental Management Linkage: A Review of the Literature. Sustainability, 10(11), 4311.

Arango, L. (2010). Importancia de los costos de la calidad y no calidad en las empresas de salud como herramienta de gestión para la competitividad. Revista EAN, 67, 75-94.

Arroyave, A. y Marulanda, F. (2019). Ecoemprendimiento, sostenibilidad y generación de valor. Revista EAN, 87, 155-172.

Bateman, T. y Snell, S. (2009). Administración. Liderazgo y colaboración en un mundo competitivo. Ciudad de México, México: McGraw-Hill.

Camisón, C., Cruz, S. y González, T. (2006). Gestión de la calidad: conceptos, enfoques, modelos y sistemas. Madrid, España: Pearson Educación.

Canossa Montes de Oca, H. (2019). Sistemas de información de marketing en la planificación estratégica de la era digital. REDMARKA. Revista de Marketing Aplicado, 23(2), 43-60.

Carlson, C. (1997). The Principles of Scientific Management by FrederickW. Taylor: the private printing. Journal of Management History, 3(1), 18-30.

Carró, F. y Caló, A. (2012). La administración científica de Frederick W. Taylor: una lectura contextualizada. En VII Jornadas de Sociología, Universidad Nacional de La Plata, Buenos Aires, Argentina.

Crosby, P.B. (1987). La calidad no cuesta. El arte de cerciorarse de la calidad. Ciudad de México, México: McGraw-Hill.

Cuatrecasas, L. (2009). Diseño avanzado de procesos y plantas de producción flexible. Técnicas de diseño y herramientas gráficas con soporte informático. Barcelona, España: Profit Editorial.

Cuatrecasas, L. (2012). Gestión de la calidad total. Organización de la producción y dirección de operaciones. Madrid, España: Ediciones Diaz de Santos.
Cuatrecasas, L. y González, J. (2017). Gestión integral de la calidad: implantación, control y certificación. Barcelona, España: Profit Editorial.

Deming, W.E. (1986). Out of the Crisis. Cambridge, USA: MIT Press.

Departamento de Estudios. (2005). Responsabilidad social empresarial alcances y potencialidades en materia laboral departamento de estudios, dirección del trabajo. Cuadernos de Investigación, 25, 2-166.

Díaz, A., Tamayo, A. y Armijos, C. (2018). Los modelos de gestión en el cumplimiento de los objetivos empresariales. Caso de Estudio: Cia. de Transportes 27 de Mayo S.A. Journal of Science and Research, 3(9), 32-40.

du Plessis, C. (2012). Towards a regenerative paradigm for the built environment. Building Research \& Information, 40(1), 7-22.

Fisher, N. (2009). Homer Sarasohn and American Involvement in the Evolution of Quality ManagementinJapan, 1945-1950. International Statistical Review, 77(2), 276-299.

Galarreta, G. (2019). Entre expectativas y percepciones. Ingnofis, 5(2).

García, P., Quispe, A. y Ráez, L. (2001). Serie de Normas NTP ISO 9000:2001. Industrial Data, $4(2), 37-42$.

González, J.L. (2017). Imaginar y crear el futuro. Madrid, España: Bubok Editorial.

Grachev, M. and Rakitsky, B. (2013). Historic horizons of Frederick Taylor's scientific management. Journal of Management History, 19(4), 512-527.

Gutiérrez, H. (2010). Calidad totaly productividad. Ciudad de México, México: McGraw-Hill.

Hahn, T. and Tampe, M. (2020). Strategies for regenerative business. Strategic Organization, 19(3), 456-477. 
Ishikawa, K. (1986). ¿Qué es el control total de calidad? La modalidad japonesa. Bogotá, Colombia: Norma.

Lee, S. (2015). The age of quality innovation. International Journal of Quality Innovation, 1(1), 1-5.

Liker, J. (2003). The Toyota Way: 14 Management Principles from the World's Greatest Manufacturer. New York, USA: McGraw-Hill Education.

Llain, S. y Hawkins, C. (2020). Cambio climático y migración forzada. Migraciones Internacionales, 11(1), 1-22.

Martínez, M. et al. (2018). Eco-etiquetado y productos verdes: desarrollo y competitividad. Tecnología en Marcha, 31(2), 87-97.

Mishraa, O., Kumar, V. and Garg, D. (2013). JIT supply chain; an investigation through general system theory. Management Science Letters, 3(3), 743-752.

Mortimer, F. et al. (2018). Sustainability in quality improvement: redefining value. Future Healthcare Journal, 5(2), 88-93.

Moscoso, K., Rojas, C. y Beraún, M. (2019). La economía circular: modelo de gestión de calidad en el Perú. PURIQ, 1(2), 189-202.

Müller, E. (2016). Desarrollo regenerativo ante el cambio global, garante de un futuro económico, social y ambiental. El caso de Centroamérica. San José, Costa Rica: Universidad para la Cooperación Internacional.

Naciones Unidas. (1987). Informe Brundtland. Nueva York, Estados Unidos: Naciones Unidas.

Omachonu, V.K., Suthummanon, S. and Einspruch, N.G. (2004). The relationship between quality and quality cost for a manufacturing company. International Journal of Quality \& Reliability Management, 21(3), 277-290.

Palominos, P. (2007). Personalización en masa: ¿una nueva forma de producción? Contribuciones Científicas y Tecnológicas, 134(1), 20-30.
Patiño, J. (2019). La cuarta revolución industrial. Ingenierías USBMed, 10(1), 1-1.

Platas, J.A.y Cervantes, M.I. (2017). Gestión integral de la calidad. Un enfoque por competencias. Ciudad de México, México: Editorial Patria.

Ponce, J., y Loor, I. (2020). Ecoeficiencia empresarial, un repaso sobre su implementación en América Latina. 593 Digital Publisher CEIT, 5(5-1), 252263.

Radjou, N. (2020). Beyond Sustainability: The Regenerative Business. Forbes. Recuperado de https://www.forbes. com/sites/naviradjou/2020/10/24/ beyond-sustainability-the-regenerativebusiness/?sh=5652c2101ab3.

Richard, A. et al. (1999). The Deming Cycle provides a framework for managing environmentally responsible process improvements. Quality Engineering, 12(2), 199-209.

Robledillo, A. y Velázquez, D. (2013). Introducción a los sistemas de gestión de la calidad total: modelo de excelencia EFQM y autoevaluación. Medicina y Seguridad del Trabajo, 59(232), 302-309.

Roland, E.y Landua, G. (2017). Empresa regenerativa. Optimizarse para la abundancia multicapital. Madrid, España: Babelcube Inc.

Siva, V. et al. (2016). The support of Quality Management to sustainable development: a literature review. Journal of Cleaner Production, 138(2), 148-157.

Summers, D. (2006). Administración de la calidad. Ciudad de México, México: Pearson Educación.

Valenzuela, F. (2016). Los costos de la mala calidad como quinto elemento del costo: aproximación teórica en la gestión de la competitividad en medio de la convergencia contable. Revista Facultad de Ciencias Económicas, 24(1), 63-84.

Valenzuela, J., Espinoza, A. y Alfaro, M. (2019). Diseño de la cadena logística inversa para modelo de negocio de economía circular. Ingeniería Industrial, 40(3), 309-318. 
Wilson, J.M. (2013). Henry Ford vs. assembly line balancing. International Journal of Production Research, 52(3), 757-765.

Yang, C. (2017). The Evolution of Quality Concepts and the Related Quality Management. En Kounis, L. (Ed.), Quality Control and Assurance. An Ancient Greek Term Re-Mastered. London, United Kingdom: IntechOpen.

Zhang, A. (2013). Quality improvement through Poka-Yoke: From engineering design to information system design. International Journal of Six Sigma and Competitive Advantage, $8(2), 147-159$.

Zhang, X. (2014). Toward a regenerative sustainability paradigm for the built environment: from vision to reality. Journal of Cleaner Production, 65(15), 3-6.

Zehir, C. and Müceldilli, B. (2012). Total Quality Management Practices' Effects on Quality Performance and Innovative Performance. Procedia-Social and Behavioral Sciences, 41, 273-280. 